\section{Case Reports in Dermatology}

\title{
Reticular Erythematous Mucinosis: Exclusive Involvement of the Axillary Region
}

\author{
Martina Burlando ${ }^{a}$ Mattia Fabio Molle ${ }^{a}$ Antonio Guadagno ${ }^{b}$ \\ Emanuele Cozzani ${ }^{a}$ Aurora Parodi ${ }^{a}$ \\ aSection of Dermatology, DISSAL, San Martino-IST Polyclinic Hospital, University of Genoa, \\ Genoa, Italy; bPathology Unit, Ospedale Policlinico San Martino, University of Genoa, \\ Genoa, Italy
}

\section{Keywords}

Mucinosis · Reticular erythematous mucinosis · Cutaneous mucinoses · Dermatitis

\begin{abstract}
Reticular erythematous mucinosis is a primary cutaneous mucinosis characterized by the presence of erythematous macules, papules, or plaques coalescing into a reticular pattern that typically involves the midline of the chest or back in middle-aged women. Because of this peculiar location, it is also called midline mucinosis. Although atypical disease localizations have been described in the literature, these have always been accompanied by midline involvement. We report a case of reticular erythematous mucinosis with exclusive involvement of the axillary region.
\end{abstract}

\section{Introduction}

Reticular erythematous mucinosis (REM) is a rare disease classified among the primary cutaneous mucinoses, although it is discussed to be a variant of lupus erythematosus tumidus because they share several clinical and histopathological similarities [1]. This condition is characterized by erythematous macules, papules, or plaques coalescing into a reticular pattern that typically involves the midline of the chest or back in middle-aged women $[2,3]$. We present a case of REM involving only the axillary regions bilaterally in an old woman.

\section{Karger $\stackrel{2}{\circ}$}




\section{Case Reports in Dermatology}
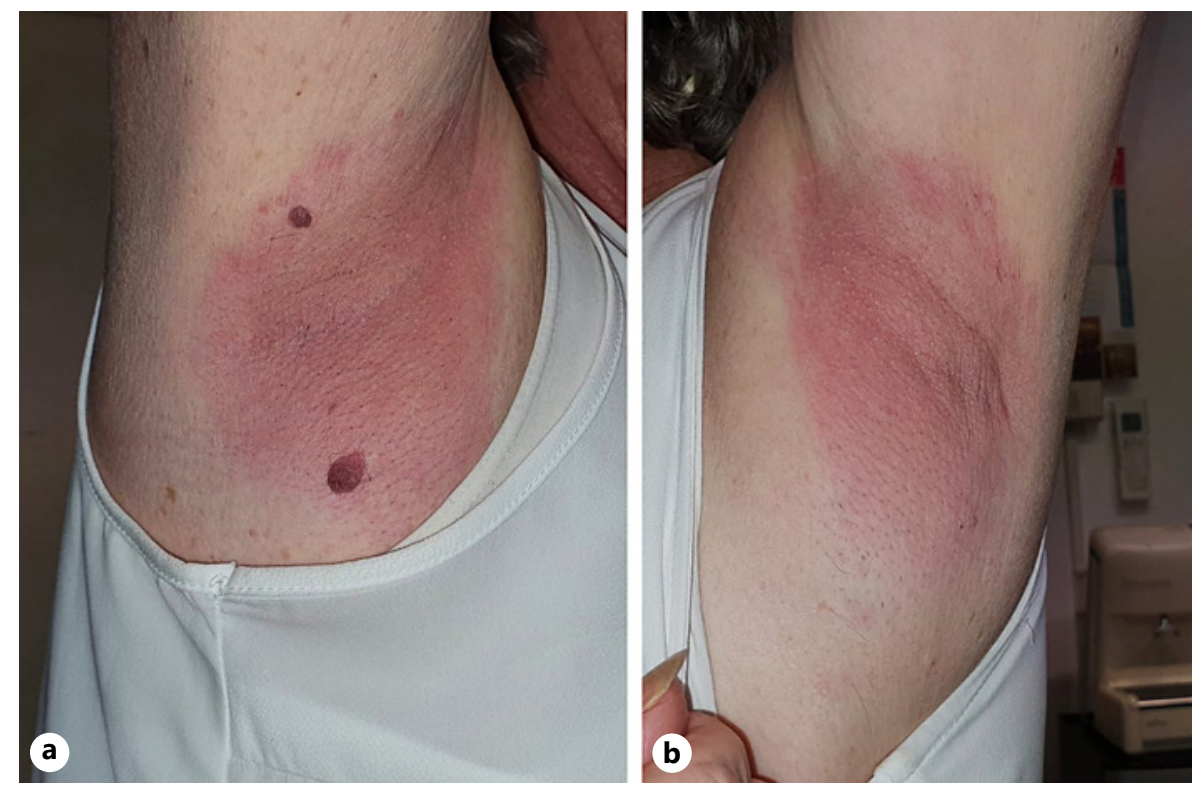

Fig. 1. a, b Erythematous patches of the axillary region bilaterally.

\section{Case Report}

A 70-year-old woman was admitted to our department with a 2-year history of asymptomatic reticular erythematous macular-papular eruption in the axillary regions with tendency to spontaneous resolution and subsequent recurrence. Clinical examination revealed a reticular erythema with the presence of some erythematous papules in the underarm area (Fig. 1a, b). Blood tests including complete blood count, lipid and biochemical profile, protein electrophoresis, liver, renal, and thyroid panel were within normal ranges. Tests for antinuclear antibodies ANA, anti-dsDNA, anti-Sm, anti-Ro/SSA, and anti-La/SSB, antiphospholipid antibodies, and complement level were also negative, and the patient did not present any other clinical features of lupus erythematous. A punch biopsy was performed, and the histopathological examination revealed in the superficial dermis a perivascular and focally perifollicular histiocytic and lymphocytic infiltrate along with focal epidermal parakeratosis with minimal spongiosis. Alcian blue-PAS staining showed a minimal amount of mucin deposition among dermal collagen bundles (Fig. 2a, b). We also performed a direct immunofluorescence of the skin which was negative for bullous diseases. On the basis of these clinical and histopathological findings, a diagnosis of REM was made. The treatment of choice in REM is antimalarial drugs such as hydroxychloroquine [4]; however, given the previous tendency to spontaneous resolution and the reluctance of the patient, we decided to avoid a systemic treatment. To date, no established alternative therapy is available. Anecdotal cases of patients treated with topical steroids, tacrolimus, or UVB phototherapy with variable results are reported in the literature $[5,6]$. Therefore, we decided to administer a topical therapy with methylprednisolone acetate $0.1 \%$ cream that led to complete remission in 3 months.

\section{Discussion}

REM is also known as midline mucinosis because it usually affects photo-exposed skin surfaces such as midline of the chest or upper midback of young and middle-aged females. The classical clinical presentation involves pink to red macules, papules, or plaques 

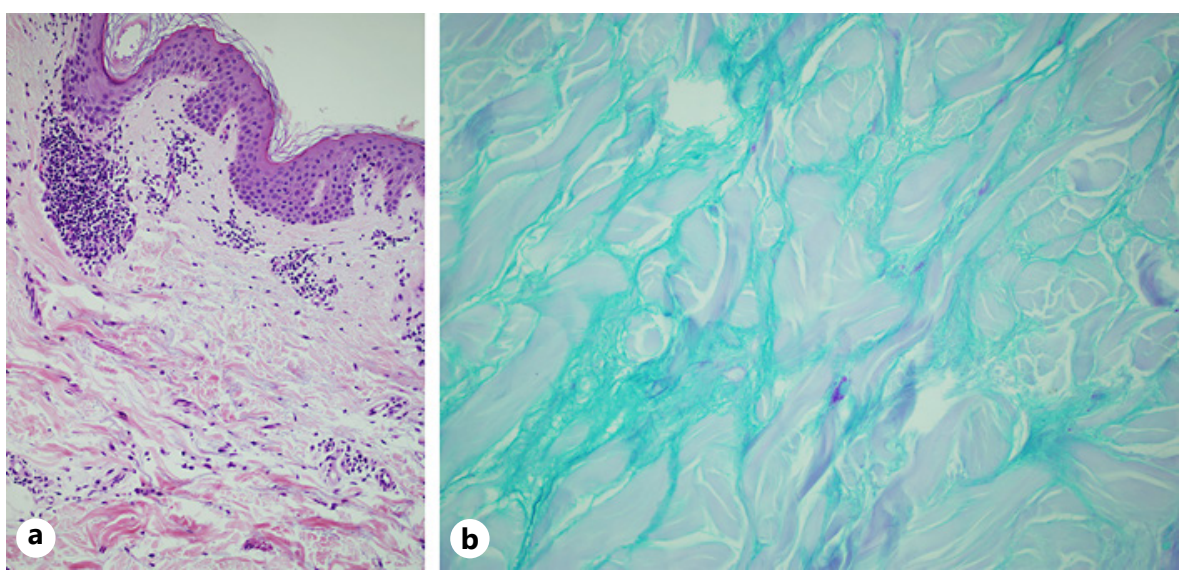

Fig. 2. a Regular epidermis and mild inflammatory infiltrate composed predominantly of lymphocytes in the dermis with a perivascular and peri-adnexal pattern (hematoxylin-eosin stain; original magnification, $\times 20$ ). b Mucin deposition in the dermis (Alcian blue-PAS stain; original magnification, $\times 40$ ).

that coalesce into a reticulated pattern. The lesions are usually asymptomatic or slightly pruritic [3].

The etiopathogenesis of REM is still undefined, although some factors have been hypothesized to play a role in the induction or exacerbation of this disease, including viral infections, immunological disturbances, or solar exposure [5]. Some conditions such as breast, lung, and colon carcinoma and hematological or hormonal dysfunction like thyroid diseases have been reported in patients with REM [7]. In this specific case, our patient in her medical history had a diagnosis of ductal lung cancer 5 years earlier for which she underwent surgical removal. The association of REM with lung cancer is particularly studied [7] because it is known that mucin synthesis is modulated by several mediators whose production is increased in the alveolar macrophages of lung cancer patients [5].

In addition to the involvement of classical areas, REM has been reported in the literature on other uncommon sites such as the face, legs, arms, abdomen, or surgical scars $[2,3,5]$. However, this involvement was accompanied by the simultaneous presence of lesions on the midline. The exclusive involvement of the axillary region is a peculiar clinical finding of this case and represents a food for thought for the clinician in the differential diagnosis of recurrent or persistent erythematous lesions especially in patients with a history of lung cancer.

\section{Statement of Ethics}

All the procedures adopted in the present study were conducted with respect to the ethical standards in the World Medical Association Declaration of Helsinki. The subject gave his written informed consent to publish the present case (including publication of images).

\section{Conflict of Interest Statement}

The authors have no conflicts of interest to declare.

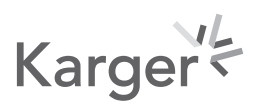




\section{Funding Sources}

The authors received no funding for this work.

\section{Author Contributions}

Study conception and design were done by M.B., M.F.M., E.C., A.G., and A.P. Acquisition of data was done by M.B., M.F.M., and A.G. Drafting of the manuscript was done by M.B., M.F.M., and A.G. Critical revision was done by E.C. and A.P.

\section{Data Availability Statement}

All data generated or analyzed during this study are included in this article and its online suppl.MaterialFiles(forallonlinesuppl.material,seewww.karger.com/doi/10.1159/000517547). Further enquiries can be directed to the corresponding author.

\section{References}

1 Cinotti E, Merlo V, Kempf W, Carli C, Kanitakis J, Parodi A, et al. Reticular erythematous mucinosis: histopathological and immunohistochemical features of 25 patients compared with 25 cases of lupus erythematosus tumidus. J Eur Acad Dermatol Venereol. 2015 Apr;29(4):689-97.

2 Atci T, Polat Ekinci A, Buyukbabani N, Baykal C. Reticular erythematous mucinosis on a mastectomy scar and relapse after mammary reconstruction. J Eur Acad Dermatol Venereol. 2017 Jan;31(1):e13-5.

3 Rongioletti F, Merlo V, Riva S, Cozzani E, Cinotti E, Ghigliotti G, et al. Reticular erythematous mucinosis: a review of patients' characteristics, associated conditions, therapy and outcome in 25 cases. Br J Dermatol. 2013 Dec;169(6):1207-11.

4 Dick J, Kroehl V, Enk AH, Toberer F. Reticular erythematous mucinosis in a young woman: complete remission following oral hydroxychloroquine. Australas J Dermatol. 2019 Nov;60(4):e343-5.

5 Thareja S, Paghdal K, Lien MH, Fenske NA. Reticular erythematous mucinosis-a review. Int J Dermatol. 2012 Aug;51(8):903-9.

6 Rubegni P, Sbano P, Risulo M, Poggiali S, Fimiani M. A case of reticular erythematous mucinosis treated with topical tacrolimus. Br J Dermatol. 2004 Jan;150(1):173-4.

7 Leon-Mateos A, Ginarte M, León L, Toribio J. Reticular erythematous mucinosis (REM) with telangiectasias associated with essential thrombocytosis and lung carcinoma. Eur J Dermatol. 2005 May-Jun;15(3):179-81.

\section{Karger's}

